\title{
Diagnostic Stewardship: A Clinical Decision Rule for Blood Cultures in Community-Onset Methicillin- Resistant Staphylococcus aureus (MRSA) Skin and Soft Tissue Infections
}

\author{
Sarah C. J. Jorgensen - Abdalhamid M. Lagnf · Sahil Bhatia • \\ Nivedita B. Singh · Laila K. Shammout - Susan L. Davis • \\ Michael J. Rybak
}

Received: January 9, 2019 / Published online: February 19, 2019

(c) The Author(s) 2019

\section{ABSTRACT}

Introduction: The emergence, spread and persistence of methicillin-resistant Staphylococcus aureus (MRSA) as a causative pathogen in community-onset (CO) skin and soft tissue infections (SSTIs) have resulted in substantial changes in the management of these infections. The indications for obtaining blood cultures in patients with CO-MRSA SSTIs remain poorly defined. The objectives of this study were to derive and validate a clinical decision rule that predicts the probability of MRSA bacteremia in CO-MRSA SSTIs and to identify a low-risk

Enhanced Digital Features To view enhanced digital features for this article go to https://doi.org/10.6084/ m9.figshare.7674074.

S. C. J. Jorgensen · A. M. Lagnf · S. Bhatia .

N. B. Singh - L. K. Shammout - S. L. Davis

M. J. Rybak ( $\bowtie)$

Anti-Infective Research Laboratory, College of Pharmacy and Health Sciences, Wayne State University, Detroit, MI, USA

e-mail:m.rybak@wayne.edu

S. L. Davis

Department of Pharmacy, Henry Ford Hospital,

Detroit, MI, USA

M. J. Rybak

Department of Pharmacy, Detroit Medical Center,

Detroit, MI, USA

M. J. Rybak

School of Medicine, Wayne State University,

Detroit, MI, USA population for whom blood cultures may be safely omitted.

Methods: This was a retrospective, case-control study with an internal temporal validation cohort conducted at two large urban academic medical centers. Hospitalized adults with COMRSA SSTI between 2010 and 2018 were included. Independent predictors of MRSA bacteremia were identified through multivariable logistic regression. A decision rule was derived using weighted coefficient-based scoring. The decision rule was validated in an internal temporal validation cohort.

Results: A total of 307 patients (155 cases and 152 controls) were included in the derivation cohort. A decision rule was created with a "major criterion" defined as purulent cellulitis and "minor criteria" defined as abnormal temperature, intravenous drug use, leukocytosis, tachycardia, body mass index $<25 \mathrm{~kg} / \mathrm{m}^{2}$ and non-upper extremity infection site. A blood culture is indicated by this rule for patients with one major or at least two minor criteria. Otherwise patients are classified as low risk, and blood cultures may be omitted. The sensitivity of the decision rule in the derivation and validation cohorts was $98.71 \%$ (95\% CI $95.42 \%$, $99.84 \%)$ and $95.65 \%$ (78.05\%, 99.89\%), respectively. The specificity was $23.03 \%(95 \%$ CI $16.59 \%, 30.54 \%)$ and $30.77 \%$ (95\% CI $24.15 \%$, $38.02 \%)$, respectively.

Conclusion: The decision rule developed and validated in this study provides a standardized, 
evidenced-based approach to determine the need for blood cultures based on bacteremia risk.

Keywords: Bloodstream infection; Methicillinresistant Staphylococcus aureus; Skin and soft tissue infection

\section{INTRODUCTION}

Skin and soft tissue infections (SSTIs) are among the most common infections encountered in both the ambulatory and inpatient settings and are associated with significant clinical and economic burdens [1, 2]. The emergence, spread and persistence of methicillin-resistant Staphylococcus aureus (MRSA) as a causative pathogen in community-onset (CO) SSTI have resulted in substantial changes to the epidemiology and management of these infections $[1,3,4]$. The changing etiology of CO-SSTIs coincided with dramatic increases in MRSA-related hospitalizations during the first decade of this century [5]. Although bacteremia has traditionally been considered a relatively rare complication of SSTI, recent evidence suggests that the MRSA USA300 clone may have an enhanced propensity to cause invasive disease, and blood culture positivity rates of $10 \%$ to $18 \%$ have been documented among hospitalized SSTI patients $[4,6,7]$. However, the general indications for obtaining blood cultures in SSTIs remain poorly defined. The high morbidity and mortality associated with MRSA bacteremia underscore the need to accurately identify patients who are at increased risk for this complication. On the other hand, indiscriminate blood culture collection has significant financial and human resource implications and results in unnecessary venipuncture [8]. Cultures that grow contaminants may result in unnecessary antimicrobial therapy, hospital admission, and extra testing and monitoring $[9,10]$. Several investigators have identified risk factors for bacteremia in patients with SSTI caused by a range of pathogens [7, 11, 12]; however no studies, to our knowledge, have focused specifically on risk factors for MRSA bacteremia in patients with CO-MRSA SSTI. To address this knowledge gap, we sought to determine sociodemographic and clinical characteristics associated with MRSA bacteremia in patients with CO-MRSA SSTI residing in the metropolitan Detroit area. We further sought to develop and validate a clinical decision rule to predict the probability of MRSA bacteremia based on characteristics readily assessable at the time of initial presentation in the emergency department (ED). This clinical tool will be particularly valuable as we enter the era of rapid diagnostics, which are capable of identifying the SSTI causative pathogen at the patient bedside.

\section{METHODS}

\section{Study Design and Setting}

A retrospective, case-control design was utilized for derivation of the clinical decision rule and an internal temporal cohort was used for validation. Both cohorts were selected from two academic medical centers servicing an urban population: the Detroit Medical Center and the Henry Ford Hospital. This study was performed in accordance with the Helsinki Declaration of 1964 and its later amendments. Approval was obtained from the Wayne State University (\#012918MP4E) and Henry Ford Hospital (\#12302) institutional review boards and the Detroit Medical Center Research Committee (\#15594) with a waiver for informed consent.

\section{Case Ascertainment}

The population of interest included adult patients ( $\geq 18$ years) who satisfied all of the following criteria: (1) admitted to inpatient services through the ED between May 2010 and May 2018; (2) International Classification of Diseases, Ninth/Tenth Revision, Clinical Modification (ICD-9/10-CM), primary diagnosis code for SSTI (abscess, cellulitis or wound infection); (3) at least three of the following signs/symptoms: local pain/tenderness, swelling, erythema, warmth, discharge, or tenderness; (4) MRSA-positive culture from skin/skin structure collected within $48 \mathrm{~h}$ of admission; (5) blood culture collected within $48 \mathrm{~h}$ of admission. 
Patients discharged directly from the ED were excluded. Blood cultures were ordered at the discretion of the treating physician, and no formal institutional guidelines were in place during the study period to guide this decision.

In the derivation cohort, a case was defined as any patient who met the above criteria and had MRSA isolated from the index blood culture for which SSTI was the suspected primary source. Patients meeting the above criteria with negative blood cultures or blood cultures deemed contaminated by the clinical microbiology laboratory served as development cohort controls. Exclusion criteria included polymicrobial bacteremia, surgical-site infections, amputations, decubitus ulcers, medical deviceassociated SSTIs, necrotizing fasciitis, osteomyelitis, septic arthritis and burns. Patients with immunocompromising conditions (hematologic malignancy; prior solid organ or bone marrow transplant; cytotoxic chemotherapy, biologic agent or antirejection agent within 30 days; functional or surgical splenectomy; HIV/AIDS; absolute neutrophil count $<500$; chronic use of systemic corticosteroids equivalent to $\geq$ prednisone $20 \mathrm{mg}$ /day $\geq 2$ weeks) were also excluded because these patients typically have a higher likelihood of poor outcomes, which demands more extensive diagnostic and aggressive management approaches irrespective of bacteremia risk [2, 13, 14].

Study patients were identified using the clinical decision support system, Theradoc (Premier Inc., Charlotte, NC, USA). A query was submitted to identify cases with both blood and skin specimen cultures positive for MRSA during the study period. A separate query was submitted to identify controls with MRSA skin specimen cultures. For the derivation cohort, the electronic medical record (EMR) of all consecutive patients meeting case criteria admitted between May 2010 and August 2016 was reviewed, whereas a random sample of patients meeting control criteria admitted during the same period was selected with the aim of including one control for every case. The validation cohort consisted of all eligible patients (MRSA SSTI \pm bacteremia) admitted between September 2016 and May 2018 to allow for estimation of bacteremia prevalence and predictive values of the clinical decision rule.

\section{Sample Size Calculation}

Abnormal vital signs have been most consistently associated with bacteremia in previous studies examining risk factors in SSTI due to a range of bacterial etiologies $[7,12,15]$. Thus, a derivation sample size of 152 cases and 152 controls was required to achieve $80 \%$ power at the 0.05 significance level to detect an odds ratio of two or greater for a risk factor with a prevalence of $25 \%$ in controls.

\section{Data Management}

Relevant patient demographic, laboratory and clinical data were extracted from the EMR retrospectively using a structured data collection form and entered into REDCap (Research Electronic Data Capture, Vanderbilt University), an electronic data capture tool hosted at Wayne State University [16]. For each vital sign and laboratory value, the most abnormal value obtained within $24 \mathrm{~h}$ of admission was extracted. Comorbidity burden was quantified by calculating the Charlson Comorbidity index [17]. Acute kidney injury (AKI) was defined as an increase in serum creatinine by 1.5 fold or $0.3 \mathrm{mg} / \mathrm{dl}$ from baseline [18]. Median household income was derived using the US Census Bureau 2012-2016 American Community Survey Estimates by zip code [19].

\section{Data Analysis and Clinical Decision Rule Development}

Baseline characteristics were evaluated using descriptive statistics. Categorical variables were compared between cases and controls using Pearson's chi-squared or Fisher's exact test. Continuous and ordinal variables were compared using the Student $t$ test and Mann-Whitney $U$ test, as appropriate. Because of the unequal proportions of bacteremic patients in the derivation (1:1 matching) and validation (true prevalence) cohorts, statistical 
comparisons between the two cohorts are not presented.

To identify independent risk factors for bacteremia, candidate variables with a $P$ value $<0.1$ and with biologic plausibility for bacteremia in the univariate analysis were entered into a multivariable logistic regression model and retained in the final model if the adjusted $P$ value was $<0.05$ using the Wald test and individually if they were retained in $\geq 95 \%$ of 1000 bootstrap samples with replacement. To develop a parsimonious model that could be used to derive a user-friendly clinical decision rule, the number of independent predictors considered for model entry was limited to ten. Model fit was assessed with the Hosmer-Lemeshow goodness-of-fit test with nonsignificant results considered adequate.

The clinical decision rule was derived using a regression coefficient-based scoring method in which independent predictive variables from the regression model were assigned a weighted value using the beta coefficients to reflect predictive power [20, 21]. Beta coefficients were adjusted for potential overfitting by applying a shrinkage factor [22]. Shrunken coefficients were divided by 0.3 and rounded to the nearest whole number $[21,23]$. The area under the receiver-operating characteristic curve (AUROC) was used to quantify the decision score's discriminative ability; an AUROC value of 0.5 denotes random predictions and a value of 1 denotes perfect discrimination. Classification and regression tree (CART) analysis were used to derive homogeneous bacteremia risk groups [24]. The performance characteristics of the score at CART-derived thresholds was evaluated by calculating the sensitivity and specificity and, for the validation cohort, the positive and negative predictive values. Statistical analyses were conducted using SPSS, version 24 (IBM Corp., Armonk, NY).

\section{RESULTS}

The derivation cohort consisted of 155 case patients matched to a random sample of 152 control patients from a pool of 5876 patients with a positive MRSA skin specimen culture.
The blood culture contamination rate in control patients was $4.6 \%$. Overall, the mean age was $48.1( \pm 17.5)$ years with a male and African American predominance $(61.2 \%$ and $59.3 \%$, respectively). The majority of patients $(65.1 \%)$ were overweight or obese [body mass index $\left.(\mathrm{BMI}) \geq 25 \mathrm{~kg} / \mathrm{m}^{2}\right]$. Over one-third (36.5\%) were considered to have a household income below the federal poverty level, and $19.9 \%$ of patients had no medical insurance. The median Charlson Comorbidity Index was 2 [interquartile range (IQR) 0, 4]. Nearly one-third (30.3\%) of patients had a pre-existing diagnosis of diabetes mellitus, while $18.2 \%$ were injection drug users (IVDU). A substantial minority of patients had been hospitalized within the previous 6 months $(20.5 \%)$, although $<10 \%$ had a recent (60-day) history of SSTI or MRSA infection.

The most frequent type of SSTI was abscess $(76.5 \%)$, and the lower extremity was the predominant site of infection (41.7\%). Most patients presented with leukocytosis (59.3\%) and $34.2 \%$ had an abnormal temperature.

As shown in Table 1, several demographic and clinical characteristics distinguished case and control patients. Bacteremic patients were significantly older, less likely to be overweight or obese, carried a higher burden of most comorbidities, had a higher rate of recent hospitalization and more often resided in a zip code area with a median household income below the federal poverty level. Case patients also presented to the ED sooner after symptom onset and had higher rates of abnormal vital signs and laboratory measures (Table 1). Purulent cellulitis and infections localized to the head and neck regions were significantly more common among case patients, whereas upper extremity infections were less often seen.

Table 2 displays the final parsimonious multivariable logistic regression model. A total of eight independent predictors for MRSA bacteremia were found; in descending order of their adjusted odds ratios, they were: purulent cellulitis, abnormal temperature, IVDU, leukocytosis, tachycardia, AKI, BMI $<25 \mathrm{~kg} / \mathrm{m}^{2}$ and non-upper extremity infection site. The AUROC of the final model was 0.859 (95\% CI 0.818, 0.901) (Fig. 1). 
Table 1 Derivation cohort baseline characteristics

\begin{tabular}{|c|c|c|c|}
\hline & $\begin{array}{l}\text { Control } \\
\text { (MRSA SSI) } \\
n=152\end{array}$ & $\begin{array}{l}\text { Case } \\
(\text { MRSA SSI + bacteremia) } \\
n=155\end{array}$ & $P$ value \\
\hline Age, years, mean $(\mathrm{SD})$ & $45.2(15.8)$ & $51.0(18.6)$ & 0.003 \\
\hline Male gender, $n(\%)$ & $87(57.2)$ & $101(65.2)$ & 0.154 \\
\hline African American race, $n(\%)$ & $85(55.9)$ & $97(62.6)$ & 0.235 \\
\hline BMI $\left(\mathrm{kg} / \mathrm{m}^{2}\right)$, median $(\mathrm{IQR})$ & $28.99(24.44,35.89)$ & $26.89(23.20,34.01)$ & 0.121 \\
\hline Overweight or obese $\left(\mathrm{BMI} \geq 25 \mathrm{~kg} / \mathrm{m}^{2}\right), n(\%)$ & $110(72.4)$ & $90(58.1)$ & 0.009 \\
\hline \multicolumn{4}{|l|}{ Annual household income level, $n(\%)^{\mathrm{a}}$} \\
\hline Poverty & $47(30.9)$ & $65(41.9)$ & 0.045 \\
\hline Low-moderate & $52(34.2)$ & $55(35.5)$ & Reference \\
\hline Middle-high & $53(34.9)$ & $35(22.6)$ & 0.017 \\
\hline \multicolumn{4}{|l|}{ Medical insurance, $n(\%)$} \\
\hline No insurance & $27(17.8)$ & $34(21.9)$ & 0.360 \\
\hline Medicare & $12(7.9)$ & $23(14.8)$ & 0.056 \\
\hline Medicaid & $39(25.7)$ & $34(21.9)$ & 0.444 \\
\hline Private/mixed & $74(48.7)$ & $64(41.3)$ & Reference \\
\hline \multicolumn{4}{|l|}{ Comorbid conditions, $n(\%)$} \\
\hline Hypertension & $49(32.2)$ & $69(44.5)$ & 0.027 \\
\hline Diabetes mellitus & $35(23.0)$ & $58(37.4)$ & 0.006 \\
\hline Chronic renal impairment & $5(3.3)$ & $22(14.2)$ & 0.001 \\
\hline IV drug abuse & $20(13.2)$ & $36(23.2)$ & 0.022 \\
\hline Charlson Comorbidity Index, median (IQR) & $1(0.3)$ & $2(2.5)$ & $<0.001$ \\
\hline \multicolumn{4}{|l|}{ MDR risk factors, $n(\%)$} \\
\hline Recent hospitalization ${ }^{\mathrm{b}}$ & $20(13.2)$ & $43(27.7)$ & 0.002 \\
\hline Recent antibiotic exposure ${ }^{c}$ & $13(8.6)$ & $16(10.3)$ & 0.596 \\
\hline Prior MRSA infection ${ }^{\mathrm{d}}$ & $7(4.6)$ & $13(8.4)$ & 0.179 \\
\hline Prior $S_{S T I}{ }^{\mathrm{d}}$ & $12(7.9)$ & $18(11.6)$ & 0.273 \\
\hline \multicolumn{4}{|l|}{ Clinical presentation $^{\mathrm{e}}, n(\%)$} \\
\hline$T<35.6{ }^{\circ} \mathrm{C}$ or $>38^{\circ} \mathrm{C}$ & $28(18.4)$ & $77(49.7)$ & $<0.001$ \\
\hline $\mathrm{HR}>100$ beats $/ \mathrm{min}$ & $52(34.2)$ & $107(69.0)$ & $<0.001$ \\
\hline $\mathrm{RR} \geq 22$ beats $/ \mathrm{min}$ & $18(11.8)$ & $43(27.7)$ & $<0.001$ \\
\hline $\mathrm{SBP} \leq 100 \mathrm{mmHg}$ & $26(17.2)$ & $46(29.7)$ & 0.009 \\
\hline WBC $>11 \times 10^{9} / 1$ & $74(48.7)$ & $108(69.7)$ & $<0.001$ \\
\hline
\end{tabular}


Table 1 continued

\begin{tabular}{lccc}
\hline & $\begin{array}{l}\text { Control } \\
\text { (MRSA SSI }) \\
n=152\end{array}$ & $\begin{array}{l}\text { Case } \\
\text { (MRSA SSI + bacteremia) } \\
n=155\end{array}$ & P value \\
\hline AKI $^{\mathrm{f}}$ & $39(25.7)$ & $75(48.4)$ & $<0.001$ \\
$2+$ SIRS criteria & $99(65.1)$ & $135(87.1)$ & $<0.001$ \\
Days from symptom onset to ED visit & $5(3.7)$ & $3(2.7)$ & 0.029 \\
median (IQR) & & & \\
SSTI type, $n$ (\%) & $140(92.1)$ & $95(61.3)$ & Reference \\
Abscess & $11(7.2)$ & $47(30.3)$ & 0.001 \\
Purulent cellulitis & $1(0.7)$ & $13(8.4)$ & 0.001 \\
Wound infection & & & Reference \\
SSTI site, $n$ (\%) & $64(42.1)$ & $64(41.3)$ & 0.025 \\
Lower extremity & $53(34.9)$ & $36(23.2)$ & 0.268 \\
Upper extremity & $17(11.2)$ & $24(15.5)$ & 0.051 \\
Trunk/torso & $18(11.8)$ & $31(20.0)$ & \\
Head/neck & & & \\
\hline
\end{tabular}

$A K I$ acute kidney injury, $B M I$ body mass index, $E D$ emergency department, $H R$ heart rate, $I Q R$ interquartile range, $I V$ intravenous, $M D R$ multidrug-resistant, $M R S A$ methicillin-resistant Staphylococcus aureus, $n$ number, $R R$ respiratory rate, $S B P$ systolic blood pressure, $S D$ standard deviation, SIRS systemic inflammatory response syndrome, SSTI skin and soft tissue infection, $T$ temperature, $W B C$ white blood cell count

a Derived using US Census data and zip code; poverty $<\$ 24,250$, low-moderate $\$ 24,251-\$ 49,999$, middle-high $\geq 50,000$

b Within 6 months

c Within 30 days

d Within 60 days

e Worst physiologic value within $24 \mathrm{~h}$ of initial presentation

${ }^{\mathrm{f}}$ Increase in serum creatinine by 1.5 or $0.3 \mathrm{mg} / \mathrm{dl}$ from baseline

The weighted coefficient-based bacteremia risk score is also shown in Table 2 . An individual patient's score is calculated by summing the applicable points, with a higher score indicating increased risk of bacteremia and total scores ranging from 0 to 31 . The discriminative ability of the score was similar to the full multivariable logistic regression model [bacteremia risk score AUROC 0.848 (95\% CI 0.806, 0.891)]. The performance characteristics of the CART-derived score thresholds are displayed in Table 3. A score threshold of 6 points resulted in a sensitivity and specificity of $98.71 \%$ (95\% CI $95.42 \%$, 99.84\%) and $23.03 \%$ (95\% CI 16.59\%, 30.54\%), respectively. Two case patients had a risk score $<$
6: (1) an 18-year-old male with no significant medical history, a temperature of $38.3{ }^{\circ} \mathrm{C}$ (3 points) and a cutaneous abscess on the right hand and (2) a previously healthy 37-year-old female with no systemic signs and an abscess on the lower left cheek (three points).

A total of 205 patients with MRSA skin/skin structure specimens satisfied the inclusion and exclusion criteria between 1 September 2016 and 15 May 2018 and were enrolled in the validation cohort. Demographic and clinical characteristics are displayed in Table 4. The prevalence of MRSA bacteremia was $11.2 \%$, and the blood culture contamination rate was $4.4 \%$. The discriminative ability of the score in the 
Table 2 Final multivariable logistic regression model for bacteremia and risk score

\begin{tabular}{|c|c|c|c|c|c|}
\hline Variable & Unadjusted OR (95\% CI) & $\begin{array}{l}\text { Adjusted OR } \\
(95 \% \mathrm{CI})\end{array}$ & $P$ value & $\beta$-Coefficient ${ }^{\mathrm{a}}$ & Points \\
\hline Purulent cellulitis & $5.578(2.763,11.264)$ & $8.449(3.465,20.603)$ & $<0.001$ & 1.979 & 7 \\
\hline$T<35.6^{\circ} \mathrm{C}$ or $>38^{\circ} \mathrm{C}$ & $4.372(2.607,7.332)$ & $4.951(2.571,9.535)$ & $<0.001$ & 1.482 & 5 \\
\hline IV drug abuse & $1.997(1.096,3.639)$ & $3.374(1.517,7.506)$ & 0.003 & 1.127 & 4 \\
\hline WBC $>11 \times 10^{9} / 1$ & $2.422(1.518,3.865)$ & $2.769(1.467,5.224)$ & 0.002 & 0.944 & 3 \\
\hline $\mathrm{HR}>100$ beats $/ \mathrm{min}$ & $4.287(2.659,6.912)$ & $2.719(1.509,4.899)$ & 0.001 & 0.927 & 3 \\
\hline Acute kidney injury & $2.716(1.679,4.396)$ & $2.570(1.345,4.914)$ & 0.004 & 0.875 & 3 \\
\hline $\mathrm{BMI}<25 \mathrm{~kg} / \mathrm{m}^{2}$ & $1.892(1.173,3.050)$ & $2.435(1.251,4.740)$ & 0.009 & 0.825 & 3 \\
\hline Non-upper extremity infection ${ }^{b}$ & $1.770(1.073,2.918)$ & $2.165(1.078,4.348)$ & 0.030 & 0.772 & 3 \\
\hline
\end{tabular}

$B M I$ body mass index, $H R$ heart rate, $I V$ intravenous, $O R$ odds ratio, $T$ temperature, $W B C$ white blood cell count

${ }^{a}$ After applying shrinkage factor $S=\left(\right.$ model $\left.X^{2}-d f\right) /$ model $X^{2}$

b Includes lower extremity, head, neck, trunk, torso

validation cohort was AUROC 0.762; 95\% CI $0.662,0.862$ (Fig. 2). As shown in Table 3, a threshold score of 6 points maintained high sensitivity (95.65\%; 95\% CI 78.05\%, 99.89\%) and further demonstrated an excellent negative predictive value $(98.25 \%$; 95\% CI $89.05 \%$, $99.74 \%)$. A clinical decision rule based on the score threshold value of 6 is outlined in Table 5 . A blood culture is indicated by this rule for patients with one major or at least two minor criteria. Otherwise, patients are classified as low risk and blood cultures may be omitted. As a practical example, blood culture collection would be indicated in a febrile patient presenting with a lower extremity cutaneous abscess by this decision rule. Blood cultures would not be indicated in an afebrile patient presenting with lower extremity cutaneous abscess and no other minor criteria.

\section{DISCUSSION}

To uncover findings that could assist clinicians in making a rapid bedside assessment of MRSA bacteremia risk in patients with CO-MRSA SSTI, we identified eight independent risk factors readily assessable at the time of initial evaluation in the ED. A predictive scoring model derived from these risk factors predicted the likelihood of bacteremia in the derivation and validation cohorts with high and moderate discriminatory ability, respectively. However, the evaluation of the clinical relevance of a scoring system is based on not only the AUC but also the clinical utility linked to that AUC. The model accurately identifies patients at low risk for bacteremia for whom blood cultures may be safely omitted. If used in our patients with CO-MRSA SSTI, the rule could appropriately reduce blood cultures by approximately $28 \%$. The clinical prediction rule may also be used to identify patients at high risk for bacteremia. Patients in the validation cohort in the highest CART-derived risk group ( $\geq 16$ points) had approximately three times the baseline population risk of bacteremia $(33.3 \%$ vs. $11.2 \%)$. A number of studies have demonstrated the benefit of early appropriate antimicrobial therapy for MRSA bacteremia [25, 26]; the prediction rule could support early initiation of more aggressive antimicrobial therapy for these higher risk patients.

To our knowledge, this is the first clinical scoring model developed specifically to determine MRSA bacteremia risk factors in CO-MRSA 


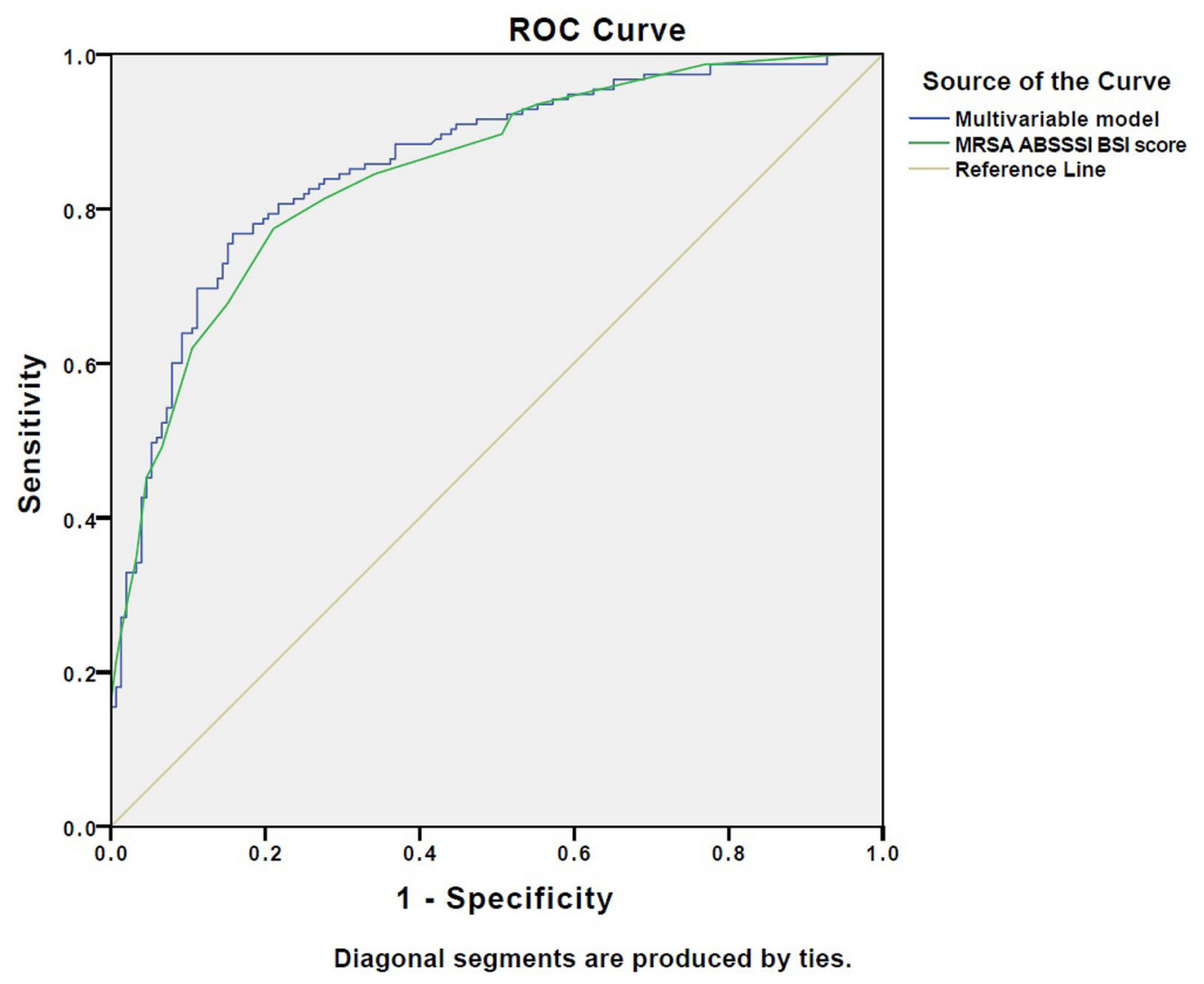

Fig. 1 Multivariable logistic regression model and bacteremia risk score area under the receiver-operator curve: derivation cohort $(n=307)$. Multivariable model

SSTI. The most recent iteration of the Infectious Diseases Society of America (IDSA) Guidelines for the management of SSTI suggest that, in immunocompetent patients such as those enrolled in the present study, blood cultures are only indicated in those with cellulitis and severe systemic signs, specifically fever or hypotension [2]. In our study, fever or hypotension was absent in $43.3 \%$ of patients with bacteremia, suggesting bacteremia may go undetected in a large proportion of patients using the IDSA criteria, possibly leading to inappropriate treatment strategies. Conversely, fever or hypotension was present in $27.3 \%$ of patients in the absence of bacteremia, representing substantial healthcare resource waste.

Many of the risk factors identified in our study, including abnormal vital signs, leukocytosis and IVDU, are consistent with those
AUROC 0.859 (95\% CI 0.818, 0.901). MRSA bacteremia risk score AUROC 0.848 (95\% CI 0.806, 0.891). Blue: multivariable model. Green: MRSA bacteremia risk score

previously reported [7, 12, 15]. However, earlier studies have included patients with SSTIs because of the wide range of pathogens, and the generalizability of results to patients with MRSA SSTI was not clear $[7,12,15]$. Purulent cellulitis was the most important risk factor in our cohort. As these infections are characterized by a diffuse spreading lesions with no defined foci amendable to source control [2], this finding should not be unexpected.

Prior studies have included patients with high burdens of underlying comorbidities, immunocompromising conditions and nosocomial SSTI $[7,12,15]$. Our study is unique in that it sought to determine risk factors for MRSA bacteremia specifically in immunocompetent adults with CO-SSTI for whom indications for blood culture collection remain uncertain. Predictive models developed in these previous 
Table 3 Performance of CART-derived risk score thresholds

\begin{tabular}{|c|c|c|c|c|c|c|}
\hline \multirow[t]{2}{*}{ Score } & \multicolumn{2}{|l|}{$\begin{array}{l}\text { Sensitivity (\%) } \\
\text { (95\% CI) }\end{array}$} & \multicolumn{2}{|l|}{$\begin{array}{l}\text { Specificity (\%) } \\
\text { (95\% CI) }\end{array}$} & \multirow{2}{*}{$\begin{array}{l}\text { Positive } \\
\text { predictive value } \\
(\%) \\
(95 \% \mathrm{CI}) \\
\text { Validation } \\
\text { cohort } \\
(n=205)\end{array}$} & \multirow{2}{*}{$\begin{array}{l}\text { Negative } \\
\text { predictive value } \\
(\%) \\
(95 \% \mathrm{CI}) \\
\text { Validation } \\
\text { cohort } \\
(n=205)\end{array}$} \\
\hline & $\begin{array}{l}\text { Derivation } \\
\text { cohort } \\
(n=307)\end{array}$ & $\begin{array}{l}\text { Validation } \\
\text { cohort } \\
(n=205)\end{array}$ & $\begin{array}{l}\text { Derivation } \\
\text { cohort } \\
(n=307)\end{array}$ & $\begin{array}{l}\text { Validation } \\
\text { cohort } \\
(n=205)\end{array}$ & & \\
\hline$\geq 6$ & $\begin{array}{c}98.71(95.42 \\
99.84)\end{array}$ & $\begin{array}{c}95.65(78.05 \\
99.89)\end{array}$ & $\begin{array}{l}23.03(16.59 \\
30.54)\end{array}$ & $\begin{array}{c}30.77(24.15 \\
38.02)\end{array}$ & $\begin{array}{c}14.86(13.29 \\
16.59)\end{array}$ & $\begin{array}{c}98.25(89.05 \\
99.74)\end{array}$ \\
\hline$\geq 12$ & $\begin{array}{c}77.42(70.02 \\
83.74)\end{array}$ & $\begin{array}{c}60.87(38.54 \\
80.29)\end{array}$ & $\begin{array}{c}78.95 \text { (71.6 } \\
85.13)\end{array}$ & $\begin{array}{c}76.92(70.11 \\
82.83)\end{array}$ & $\begin{array}{c}25.0(17.94 \\
33.69)\end{array}$ & $\begin{array}{c}93.96(90.28 \\
96.30)\end{array}$ \\
\hline$\geq 16$ & $\begin{array}{l}45.16(37.17 \\
53.35)\end{array}$ & $\begin{array}{l}34.78(16.38, \\
57.27)\end{array}$ & $\begin{array}{l}95.39(90.74 \\
98.13)\end{array}$ & $\begin{array}{l}91.21(86.12, \\
94.89)\end{array}$ & $\begin{array}{l}33.33(19.42 \\
50.91)\end{array}$ & $\begin{array}{l}91.71(89.11, \\
93.74)\end{array}$ \\
\hline
\end{tabular}

studies have also included laboratory measures not routinely collected in CO-SSTI patients (i.e., albumin, lactate and c-reactive protein) $[7,12,15]$, limiting practical utility in the ED.

We observed that bacteremic patients were more likely to reside in areas of Detroit where the median household income fell below the federal poverty level on univariate analysis. Many studies have found that persons living in areas of lower socioeconomic status (SES) have higher rates of MRSA colonization and infection and, specifically, higher rates of invasive community-associated MRSA infection [27-29]. SES may impact infectious disease rates and outcomes through differences in living conditions, comorbidities, heath literacy and behaviors such as smoking and IVDU [29]. Interestingly, we observed that bacteremic patients sought care sooner after symptom onset, suggesting that access to medical care was not a driver of SES disparities. SES did not remain an independent predictor in multivariable analysis, likely because covariates included in our model may also be on the causal pathway linking SES to bacteremia. It is possible that the relationships between other risk factors and SES differ in areas outside of metropolitan Detroit, resulting in alternative risk models. A broader understanding of the mechanisms by which SES influences complications in CO-MRSA SSTIs would be a major step forward in developing strategies to reduce the public health burden of SSTIs.

A notable finding of our study was that excess weight appeared to have a protective effect against progression to MRSA bacteremia. Obesity is a well-described risk factor for MRSA colonization and acquisition of a number of infectious diseases including SSTIs [30, 31]. However, data are conflicting regarding whether this predisposition toward acquiring infection also translates into worse outcomes in patients who become infected [32-36]. A growing body of evidence now supports the "obesity paradox," the notion that, as observed in the present study, obesity confers a protective effect against complications in certain infections [35-37]. One hypothesis is that that the chronic inflammatory state associated with obesity may actually lead to a heightened state of host immune defense in certain infections [38]. Emerging evidence in $S$. aureus bacteremia has uncovered the prognostic importance of a dysregulated immune response [39, 40]. Further studies untangling the complex relationship among bodyweight, host immune response and outcomes are needed.

Our study has several important limitations to consider. First, the differing case mix in the development and validation cohorts meant that we could not perform meaningful direct 
Table 4 Validation cohort baseline characteristics

\begin{tabular}{|c|c|}
\hline Characteristic & $\begin{array}{l}n(\%) \\
n=205\end{array}$ \\
\hline Age, years, mean (SD) & $43.53(15.38)$ \\
\hline Male gender, $n(\%)$ & $111(54.1)$ \\
\hline African American race & $126(61.5)$ \\
\hline $\operatorname{BMI}\left(\mathrm{kg} / \mathrm{m}^{2}\right)$, median $(\mathrm{IQR})$ & $27.27(23.06,32.96)$ \\
\hline $\begin{array}{l}\text { Overweight or obese (BMI } \\
\left.\geq 25 \mathrm{~kg} / \mathrm{m}^{2}\right), n(\%)\end{array}$ & $132(64.4)$ \\
\hline \multicolumn{2}{|c|}{ Annual household income level, $n(\%)^{\mathrm{a}}$} \\
\hline Poverty & $91(44.4)$ \\
\hline Low-moderate & $77(37.6)$ \\
\hline Middle-high & $32(15.6)$ \\
\hline \multicolumn{2}{|l|}{ Medical insurance, $n(\%)$} \\
\hline No insurance & $17(8.3)$ \\
\hline Medicare & $18(8.8)$ \\
\hline Medicaid & $48(23.4)$ \\
\hline Private/mixed & $122(59.5)$ \\
\hline \multicolumn{2}{|l|}{ Comorbid conditions, $n$ (\%) } \\
\hline Hypertension & $60(29.3)$ \\
\hline Diabetes mellitus & $33(16.1)$ \\
\hline Chronic renal impairment & $7(3.4)$ \\
\hline IV drug abuse & $36(17.6)$ \\
\hline $\begin{array}{l}\text { Charlson Comorbidity Index, } \\
\text { median (IQR) }\end{array}$ & $1(0,2)$ \\
\hline \multicolumn{2}{|l|}{ MDR risk factors, $n(\%)$} \\
\hline Recent hospitalization ${ }^{\mathrm{b}}$ & $51(24.9)$ \\
\hline Recent antibiotic exposure ${ }^{c}$ & $31(15.1)$ \\
\hline Prior MRSA infection ${ }^{\mathrm{d}}$ & $5(2.4)$ \\
\hline Prior SSTI & $18(8.8)$ \\
\hline \multicolumn{2}{|l|}{ Clinical presentation $^{\mathrm{e}}, n(\%)$} \\
\hline$T<35.6^{\circ} \mathrm{C}$ or $>38^{\circ} \mathrm{C}$ & $22(10.7)$ \\
\hline $\mathrm{HR}>100$ beats $/ \mathrm{min}$ & $82(40.0)$ \\
\hline $\mathrm{RR} \geq 22$ beats $/ \mathrm{min}$ & $27(13.2)$ \\
\hline $\mathrm{SBP} \leq 100 \mathrm{mmHg}$ & $44(21.5)$ \\
\hline
\end{tabular}

Table 4 continued

\begin{tabular}{lc}
\hline Characteristic & $\begin{array}{l}n(\%) \\
n=205\end{array}$ \\
\hline WBC $>11 \times 10^{9} / 1$ & $94(45.9)$ \\
AKI $^{\mathrm{f}}$ & $25(12.2)$ \\
$2+$ SIRS criteria & $131(63.9)$ \\
Days from symptom onset to ED visit, & $4(2.7)$ \\
median (IQR) & \\
SSTI type, $n$ (\%) & \\
Abscess & $153(74.6)$ \\
Purulent cellulitis & $35(17.1)$ \\
Wound infection & $17(8.3)$ \\
SSTI site, $n$ (\%) & \\
Lower extremity & $72(35.1)$ \\
Upper extremity & $78(38.0)$ \\
Trunk/torso & $33(16.1)$ \\
Head/neck & $22(10.7)$ \\
\hline
\end{tabular}

$A K I$ acute kidney injury, $B M I$ body mass index, $E D$ emergency department, $H R$ heart rate, $I Q R$ interquartile range, $I V$ intravenous, $M D R$ multidrug-resistant, MRSA methicillin-resistant Staphylococcus aureus, $n$ number, $R R$ respiratory rate, $S B P$ systolic blood pressure, $S D$ standard deviation, SIRS systemic inflammatory response syndrome, SSTI skin and soft tissue infection, $T$ temperature, $W B C$ white blood cell count

${ }^{a}$ Derived using US Census data and zip code; poverty $<\$ 24,250$, low-moderate $\$ 24,251-\$ 49,999$, middle-high $\geq 50,000$

${ }^{\mathrm{b}}$ Within 6 months

c Within 30 days

${ }^{\mathrm{d}}$ Within 60 days

e Worst physiologic value within $24 \mathrm{~h}$ of initial presentation

${ }_{\mathrm{f}}^{\mathrm{f}}$ Increase in serum creatinine by 1.5 fold or $0.3 \mathrm{mg} / \mathrm{dl}$ from baseline

comparisons of baseline demographic and clinical characteristics. However, our approach enabled us to evaluate a larger number of risk factors and perform more robust statistical analysis while still obtaining an estimate of blood culture positivity prevalence and the 


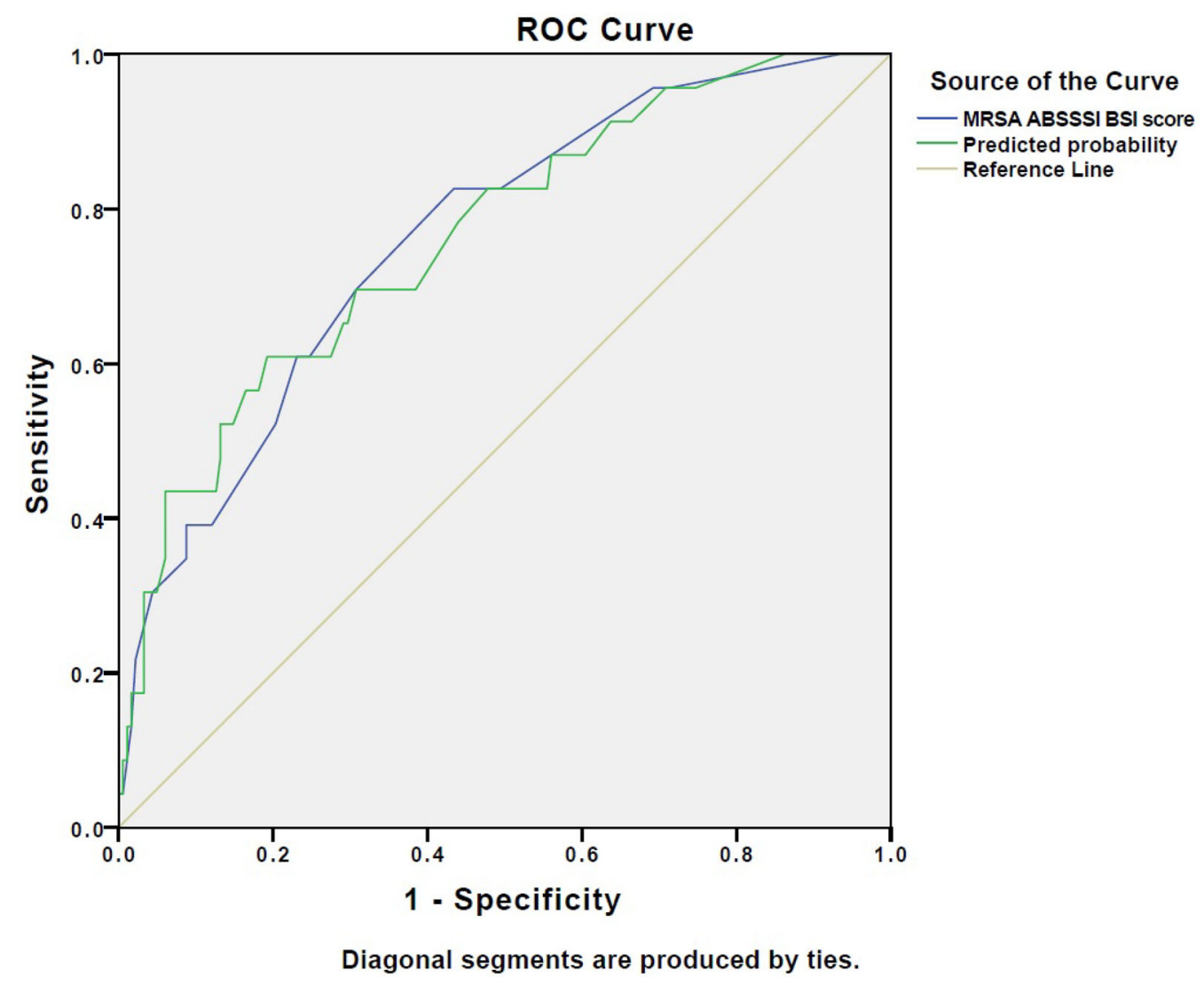

Fig. 2 Multivariable logistic regression model and bacteremia risk score area under the receiver-operator curve: validation cohort $(n=205)$. Multivariable model

Table 5 Proposed blood culture decision rule in patients with MRSA SSTI

\begin{tabular}{ll}
\hline Major criterion & Minor criteria \\
\hline Purulent cellulitis & History of IV drug use \\
& BMI $<25 \mathrm{~kg} / \mathrm{m}^{2}$ \\
& Non-upper extremity infection site \\
& Acute kidney injury \\
& Temperature $<35.6{ }^{\circ} \mathrm{C}$ or $>38^{\circ} \mathrm{C}$ \\
& WBC $>11 \times 10^{9} / 1$ \\
& Heart rate $>100$ beats $/ \mathrm{min}$ \\
\hline
\end{tabular}

Blood cultures should be strongly considered in patients with 1 major criterion or $\geq 2$ minor criteria

$B M I$ body mass index, $I V$ intravenous, $W B C$ white blood cell count
AUROC 0.763 (95\% CI 0.658, 0.868). MRSA bacteremia risk score AUROC 0.762 (95\% CI 0.662, 0.862). Blue: multivariable model. Green: MRSA bacteremia risk score

potential impact of operationalizing our findings. Second, the study is subject to the inherent bias of its retrospective design. Blood cultures were performed at the discretion of the treating physician, and previous studies have clearly demonstrated that physicians are more inclined to order blood cultures in patients with a more severe presentation [7, 12, 41]. Additionally, we focused on SSTIs severe enough to require inpatient admission, and thus our findings may not generalize to patients with COSSTI managed in the outpatient setting. Although MRSA is now the predominant pathogen in purulent SSTIs in many regions, in the absence of rapid diagnostics, the true causative pathogen is not known at the point when blood cultures are ordered. Our data were obtained from two medical centers serving a predominantly inner city population of lower 
socioeconomic status and may not be generalizable to other institutions in distinct geographic areas or serving a different patient population by sociodemographic factors. Validation in other populations is necessary before widespread clinical use. Finally, infection management decisions are complex activities requiring the clinician to weigh a number of competing risks and benefits based on the probabilities of disease and the quality of data available. The decision rule developed by our group is an attractive strategy to assist in this decision-making process but should never supersede clinical judgment.

\section{CONCLUSION}

Based on demographic and clinical characteristics, the decision rule developed and validated in this study provides a practical tool to stratify patients with CO-MRSA SSTI based on bacteremia risk and assist in clinical decision-making pertaining to the utility of blood cultures.

\section{ACKNOWLEDGEMENTS}

\section{Michael J. Rybak is Editor in Chief of Infectious Diseases and Therapy.}

Funding. No funding or sponsorship was received for this study or publication of this article.

Authorship. All named authors meet the International Committee of Medical Journal Editors (ICMJE) criteria for authorship for this article, take responsibility for the integrity of the work as a whole and have given their approval for this version to be published.

Prior Presentation. These data were presented in part at ASM Microbe 2018, Atlanta, GA, on 9 June 2018 (Abstract no. 687).

Disclosures. Sarah C.J. Jorgensen, Abdalhamid M. Lagnf, Sahil Bhatia, Nivedita B. Singh, Laila K. Shammout, Susan L. Davis and Michael J. Rybak have nothing to disclose.
Compliance with Ethics Guidelines. This study was performed in accordance with the Helsinki Declaration of 1964 and its later amendments. Approval was obtained from the Wayne State University (\#012918MP4E) and Henry Ford Hospital (\#12302) institutional review boards and the Detroit Medical Center Research Committee (\#15594) with a waiver for informed consent.

Data Availability. The data sets during and/ or analyzed during the current study are available from the corresponding author on reasonable request.

Open Access. This article is distributed under the terms of the Creative Commons Attribution-NonCommercial 4.0 International License (http://creativecommons.org/licenses/ by-nc/4.0/), which permits any noncommercial use, distribution, and reproduction in any medium, provided you give appropriate credit to the original author(s) and the source, provide a link to the Creative Commons license, and indicate if changes were made.

\section{REFERENCES}

1. Pollack CV Jr, Amin A, Ford WT Jr, et al. Acute bacterial skin and skin structure infections (ABSSSI): practice guidelines for management and care transitions in the emergency department and hospital. J Emerg Med. 2015;48:508-19.

2. Stevens DL, Bisno AL, Chambers HF, et al. Practice guidelines for the diagnosis and management of skin and soft tissue infections: 2014 update by the Infectious Diseases Society of America. Clin Infect Dis. 2014;59:e10-52.

3. Hersh AL, Chambers HF, Maselli JH, et al. National trends in ambulatory visits and antibiotic prescribing for skin and soft-tissue infections. Arch Intern Med. 2008;168:1585-91.

4. Jenkins TC, Sabel AL, Sarcone EE, et al. Skin and soft-tissue infections requiring hospitalization at an academic medical center: opportunities for antimicrobial stewardship. Clin Infect Dis. 2010;51:895-903.

5. Klein E, Smith DL, Laxminarayan R. Hospitalizations and deaths caused by methicillin-resistant 
Staphylococcus aureus, United States, 1999-2005. Emerg Infect Dis. 2007;13:1840-6.

6. Suaya JA, Eisenberg DF, Fang C et al. Skin and soft tissue infections and associated complications among commercially insured patients aged 0-64 years with and without diabetes in the US. PLoS One. 2013;8:e60057.

7. van Daalen FV, Kallen MC, van den Bosch CMA, et al. Clinical condition and comorbidity as determinants for blood culture positivity in patients with skin and soft-tissue infections. Eur J Clin Microbiol Infect Dis. 2017;36:1853-8.

8. Miller JM, Binnicker MJ, Campbell S et al. A guide to utilization of the microbiology laboratory for diagnosis of infectious diseases: 2018 update by the Infectious Diseases Society of America and the American Society for Microbiology. Clin Infect Dis. 2018;67:e1-94.

9. Bates DW, Goldman L, Lee TH. Contaminant blood cultures and resource utilization. The true consequences of false-positive results. JAMA. 1991;265:365-9.

10. Surdulescu S, Utamsingh D, Shekar R. Phlebotomy teams reduce blood-culture contamination rate and save money. Clin Perform Qual Health Care. 1998;6:60-2.

11. Lee J, Hwang SS, Kim K, et al. Bacteremia prediction model using a common clinical test in patients with community-acquired pneumonia. Am J Emerg Med. 2014;32:700-4.

12. Lipsky BA, Kollef MH, Miller LG, et al. Predicting bacteremia among patients hospitalized for skin and skin-structure infections: derivation and validation of a risk score. Infect Control Hosp Epidemiol. 2010;31:828-37.

13. Micek ST, Hoban AP, Pham V, et al. Bacteremia increases the risk of death among patients with softtissue infections. Surg Infect (Larchmt). 2010;11:169-76.

14. Pulido-Cejudo A, Guzman-Gutierrez M, JalifeMontano A, et al. Management of acute bacterial skin and skin structure infections with a focus on patients at high risk of treatment failure. Ther Adv Infect Dis. 2017;4:143-61.

15. Lee CY, Kunin CM, Chang C, et al. Development of a prediction model for bacteremia in hospitalized adults with cellulitis to aid in the efficient use of blood cultures: a retrospective cohort study. BMC Infect Dis. 2016;16:581.

16. Harris PA, Taylor R, Thielke R, et al. Research electronic data capture (REDCap)-a metadata-driven methodology and workflow process for providing translational research informatics support. J Biomed Inform. 2009;42:377-81.

17. Charlson ME, Pompei P, Ales KL, et al. A new method of classifying prognostic comorbidity in longitudinal studies: development and validation. J Chronic Dis. 1987;40:373-83.

18. Mehta RL, Kellum JA, Shah SV, et al. Acute Kidney Injury Network: report of an initiative to improve outcomes in acute kidney injury. Crit Care. 2007;11:R31.

19. United States Census Bureau. 2012-2016 American Community Survey 5-Year Estimates. https:// factfinder.census.gov. Last accessed 12/2/2017.

20. Moons KG, Harrell FE, Steyerberg EW. Should scoring rules be based on odds ratios or regression coefficients? J Clin Epidemiol. 2002;55:1054-5.

21. Sullivan LM, Massaro JM, D'Agostino RB Sr. Presentation of multivariate data for clinical use: the Framingham Study risk score functions. Stat Med. 2004;23:1631-60.

22. Van Houwelingen JC, Le Cessie S. Predictive value of statistical models. Stat Med. 1990;9:1303-25.

23. Moons KG, Donders AR, Steyerberg EW, et al. Penalized maximum likelihood estimation to directly adjust diagnostic and prognostic prediction models for overoptimism: a clinical example. J Clin Epidemiol. 2004;57:1262-70.

24. Zhang H, Burthon S. Recursive partitioning in the health sciences. New York: Springer; 1999.

25. Lodise TP, McKinnon PS, Swiderski L, et al. Outcomes analysis of delayed antibiotic treatment for hospital-acquired Staphylococcus aureus bacteremia. Clin Infect Dis. 2003;36:1418-23.

26. Schramm GE, Johnson JA, Doherty JA, et al. Methicillin-resistant Staphylococcus aureus sterilesite infection: the importance of appropriate initial antimicrobial treatment. Crit Care Med. 2006;34:2069-74.

27. Herman RA, Kee VR, Moores KG, et al. Etiology and treatment of community-associated methicillin-resistant Staphylococcus aureus. Am J Health Syst Pharm. 2008;65:219-25.

28. See I, Wesson P, Gualandi N, et al. Socioeconomic factors explain racial disparities in invasive community-associated methicillin-resistant Staphylococcus aureus disease rates. Clin Infect Dis. 2017;64:597-604. 
29. Tong SY, van Hal SJ, Einsiedel L, et al. Impact of ethnicity and socio-economic status on Staphylococcus aureus bacteremia incidence and mortality: a heavy burden in indigenous Australians. BMC Infect Dis. 2012;12:249.

30. Falagas ME, Kompoti M. Obesity and infection. Lancet Infect Dis. 2006;6:438-46.

31. Huttunen R, Syrjanen J. Obesity and the risk and outcome of infection. Int $\mathrm{J}$ Obes (Lond). 2013;37:333-40.

32. Falagas ME, Athanasoulia AP, Peppas G, et al. Effect of body mass index on the outcome of infections: a systematic review. Obes Rev. 2009;10:280-9.

33. Huttunen R, Laine J, Lumio J, et al. Obesity and smoking are factors associated with poor prognosis in patients with bacteraemia. BMC Infect Dis. 2007;7:13.

34. Longo C, Bartlett G, Macgibbon B, et al. The effect of obesity on antibiotic treatment failure: a historical cohort study. Pharmacoepidemiol Drug Saf. 2013;22:970-6.

35. Singanayagam A, Singanayagam A, Chalmers JD. Obesity is associated with improved survival in community-acquired pneumonia. Eur Respir J. 2013;42:180-7.
36. Wacharasint P, Boyd JH, Russell JA, et al. One size does not fit all in severe infection: obesity alters outcome, susceptibility, treatment, and inflammatory response. Crit Care. 2013;17:R122.

37. Roth J, Sahota N, Patel P et al. Obesity paradox, obesity orthodox, and the metabolic syndrome: an approach to unity. Mol Med. 2016;22:873-885.

38. Hsu A, Aronoff DM, Phipps J, et al. Leptin improves pulmonary bacterial clearance and survival in ob/ ob mice during pneumococcal pneumonia. Clin Exp Immunol. 2007;150:332-9.

39. Minejima E, Bensman J, She RC, et al. A dysregulated balance of proinflammatory and anti-inflammatory host cytokine response early during therapy predicts persistence and mortality in Staphylococcus aureus bacteremia. Crit Care Med. 2016;44:671-9.

40. Rose WE, Eickhoff JC, Shukla SK, et al. Elevated serum interleukin-10 at time of hospital admission is predictive of mortality in patients with Staphylococcus aureus bacteremia. J Infect Dis. 2012;206:1604-11.

41. Shapiro NI, Wolfe RE, Wright SB, et al. Who needs a blood culture? A prospectively derived and validated prediction rule. J Emerg Med. 2008;35:255-64. 